Supporting Information

\title{
Blending Behavior of High-Degree Long-Chain-Branched \\ Polypropylene Prepared by Ziegler-Natta Catalysis with Common Polypropylene
}

Kang Li $,{ }^{\dagger},+$ Yawei Qin,${ }^{\dagger}$ Songmei Zhao,${ }^{\dagger}$ Jin-Yong Dong ${ }^{\dagger, t, *}$

$\dagger$ CAS Key Laboratory of Engineering Plastics, Institute of Chemistry, Chinese

Academy of Sciences, Beijing 100190, China

$\$$ University of Chinese Academy of Sciences, Beijing 100049, China

*Corresponding author: jydong@iccas.ac.cn 
Table S1. Results of Blending of LCB-PPH and PPH

\begin{tabular}{ccccc}
\hline Entry & $\begin{array}{c}\text { LCB- } \\
\text { PPH/PPH } \\
(\mathrm{w} / \mathrm{w})\end{array}$ & $g^{\prime}<\min >$ & $\begin{array}{c}M_{\mathrm{w}} \\
\left(10^{4} \mathrm{~g} / \mathrm{mol}\right)\end{array}$ & $Ð$ \\
\hline LCB-PPH & $100 / 0$ & 0.70 & 52.0 & 8.7 \\
PPH & $0 / 100$ & 1.0 & 37.4 & 5.9 \\
B1 & $20 / 80$ & 0.83 & 43.8 & 6.9 \\
B2 & $30 / 70$ & 0.79 & 44.8 & 7.3 \\
B3 & $40 / 60$ & 0.79 & 45.0 & 7.3 \\
B4 & $50 / 50$ & 0.77 & 46.5 & 7.7 \\
B5 & $60 / 40$ & 0.76 & 46.5 & 7.6 \\
B6 & $70 / 30$ & 0.75 & 47.0 & 7.8 \\
B7 & $80 / 20$ & 0.74 & 48.1 & 8.3
\end{tabular}


Table S2. Results of Blending of LCB-PPR and PPR

\begin{tabular}{ccccc}
\hline Entry & $\begin{array}{c}\text { LCB- } \\
\text { PPR/PPR } \\
(\mathrm{w} / \mathrm{w})\end{array}$ & $g^{\prime}{ }^{\prime}$ min $>$ & $\begin{array}{c}M_{\mathrm{w}} \\
\left(10^{4} \mathrm{~g} / \mathrm{mol}\right)\end{array}$ & $Ð$ \\
\hline LCB-PPR & $100 / 0$ & 0.66 & 50.1 & 10.0 \\
PPR & $0 / 100$ & 1.0 & 37.8 & 5.3 \\
B8 & $20 / 80$ & 0.83 & 41.4 & 6.6 \\
B9 & $30 / 70$ & 0.80 & 42.7 & 7.3 \\
B10 & $40 / 60$ & 0.79 & 43.2 & 7.4 \\
B11 & $50 / 50$ & 0.78 & 44.3 & 8.0 \\
B12 & $60 / 40$ & 0.75 & 45.8 & 8.4 \\
B13 & $70 / 30$ & 0.74 & 47.9 & 8.7 \\
B14 & $80 / 20$ & 0.71 & 48.3 & 9.3
\end{tabular}


Table S3. Melt Strength, Maximum Drawing Velocity, and Melt Flow Rate Data for Blends of LCB-PPH and PPH

\begin{tabular}{ccccc}
\hline Entry & $\begin{array}{c}\text { LCB-PPH/PPH } \\
(\mathrm{w} / \mathrm{w})\end{array}$ & $\begin{array}{c}\left.\mathrm{MS}{ }^{a}\right) \\
(\mathrm{cN})\end{array}$ & $\begin{array}{c}\left.\mathrm{MDV}^{b}\right) \\
\left({ }^{\circ} \mathrm{C}\right)\end{array}$ & $\begin{array}{c}\left.\mathrm{MFR}^{c}\right) \\
(\mathrm{g} / 10 \mathrm{~min})\end{array}$ \\
\hline LCB-PPH & $100 / 0$ & 58.8 & 225 & 0.3 \\
PPH & $0 / 100$ & 8.2 & 220 & 2.4 \\
B1 & $20 / 80$ & 19.3 & 250 & 1.5 \\
B2 & $30 / 70$ & 22.2 & 250 & 1.3 \\
B3 & $40 / 60$ & 30.3 & 243 & 1.2 \\
B4 & $50 / 50$ & 34.4 & 243 & 0.9 \\
B5 & $60 / 40$ & 37.5 & 237 & 0.8 \\
B6 & $70 / 30$ & 44.1 & 230 & 0.6 \\
B7 & $80 / 20$ & 46.6 & 225 & 0.5 \\
${ }^{a}$ Melt strength; ${ }^{b}$ Maximum drawing velocity; ${ }^{c}$ Melt flow rate. &
\end{tabular}


Table S4. Melt Strength, Maximum Drawing Velocity, and Melt Flow Rate Data for Blends of LCB-PPR and PPR

\begin{tabular}{ccccc}
\hline Entry & $\begin{array}{c}\text { LCB- } \\
\text { PPR/PPR } \\
(\mathrm{w} / \mathrm{w})\end{array}$ & $\begin{array}{c}\left.\text { MS }{ }^{a}\right) \\
(\mathrm{cN})\end{array}$ & $\begin{array}{c}\text { MDV } \\
\left({ }^{\circ} \mathrm{C}\right)\end{array}$ & $\begin{array}{c}\text { MFR }) \\
(\mathrm{g} / 10 \mathrm{~min})\end{array}$ \\
\hline LCB-PPR & $100 / 0$ & 42.2 & 225 & 0.5 \\
PPR & $0 / 100$ & 8.2 & 231 & 2.4 \\
B8 & $20 / 80$ & 18.9 & 265 & 1.3 \\
B9 & $30 / 70$ & 22.4 & 260 & 1.1 \\
B10 & $40 / 60$ & 29.3 & 250 & 0.9 \\
B11 & $50 / 50$ & 33.0 & 243 & 0.8 \\
B12 & $60 / 40$ & 34.2 & 237 & 0.7 \\
B13 & $70 / 30$ & 35.7 & 231 & 0.6 \\
B14 & $80 / 20$ & 39.5 & 228 & 0.6 \\
${ }^{a}$ Melt strength; ${ }^{b}$ Maximum drawing velocity; ${ }^{c}$ Melt flow rate. &
\end{tabular}

